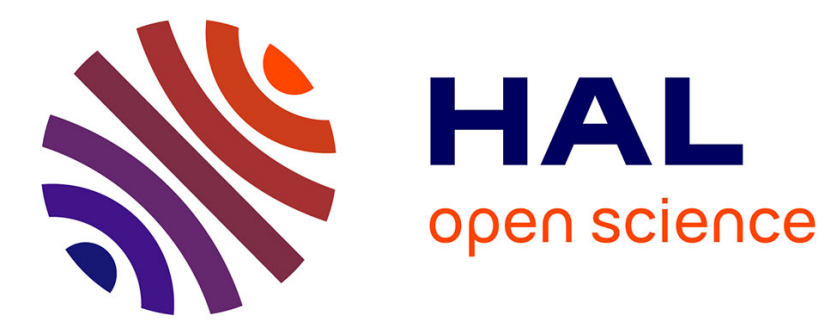

\title{
De la maison-mon(a)de à la ville-maison
}

Daniel Pinson D. Pinson

\section{To cite this version:}

Daniel Pinson D. Pinson. De la maison-mon(a)de à la ville-maison. Communications, 2002, Manières d'habiter, 73, pp.217-231. hal-00789313

\section{HAL Id: hal-00789313 https://hal.science/hal-00789313}

Submitted on 18 Feb 2013

HAL is a multi-disciplinary open access archive for the deposit and dissemination of scientific research documents, whether they are published or not. The documents may come from teaching and research institutions in France or abroad, or from public or private research centers.
L'archive ouverte pluridisciplinaire HAL, est destinée au dépôt et à la diffusion de documents scientifiques de niveau recherche, publiés ou non, émanant des établissements d'enseignement et de recherche français ou étrangers, des laboratoires publics ou privés. 
DE LA MAISON-MON(A)DE A LA VILLE-MAISON

Par Daniel Pinson

Paru dans Communications, revue du Centre d'études transdisciplinaires de l'EHESS (Dir. Edgar Morin), nº73, Manières d'habiter (ed. Ph Bonnin), Paris, Seuil, 2002, p. 217-231.

L'habitation a souvent été étudiée pour elle-même, ou encore dans l'ensemble immédiat dont elle fait partie : l'immeuble, la cité ou le village. On s'intéressera ici aux territoires de la maison d'écart, à ces itinéraires et ces contrées que l'habitant, sorti de la ville tout en y gardant son emploi, parcourt de manière contrainte ou choisie. Ces chemins et ces destinations, connotés de valeurs positives ou négatives, qui les instituent ainsi en " lieux " et en " non-lieux ", contribuent à la construction d'aires urbaines de pratiques qui peuvent former, par la familiarité de leur fréquentation et l'intensité de leur appropriation, des "territoires ".

La maison ici étudiée est celle des "campagnes urbaines ", régions autrefois rurales, soumises à la pression métropolitaine et gagnées par une urbanisation diffuse. Ses principes, autrefois inscrits dans les POS, consistent à la limiter, en n'autorisant les constructions que sur des parcelles de superficie importante (entre 4.000 et $10.000 \mathrm{~m} 2$ ).

Le phénomène dit de " l'étalement urbain " marque l'évolution des villes depuis le dernier quart du siècle passé. L'installation lointaine de ceux dont l'activité rémunérée est située en ville consolide ou parachève un processus de mise à distance de la résidence et du lieu de travail qui n'a été rendu possible à cette échelle que par la généralisation de l'auto-mobilité.

Au cours de ce processus une nouvelle figure de l'habitation se construit : elle partage, sous l'effet des transformations des modes de vie et de consommation, bien des traits de l'habiter d'aujourd'hui et du logement actuel. Mais elle les porte aussi à leur paroxysme, vers des manifestations extrêmes. L'une de ses traductions réside dans une relation organique à la ville plus ténue que ne le laisserait penser la décision d'éloignement en " campagne urbaine ". C'est l'étude de ce paradoxe que nous souhaitons aborder dans cet article. La matière première qui le nourrit chemine par des références, des travaux successifs et des voisinages intellectuels nombreux et persévérants quant à la voie qu'ils ont empruntée. Une recherche récente portant sur la maison du péri-urbain dans l'aire métropolitaine Aixo-marseillaise étudie plus amplement ce mode résidentiel et nourrira largement le présent article.

\section{Une maison plus retirée et en même temps plus ouverte au monde (la maison-mon(a)de)}

L'éloignement de la ville est pour partie motivé par la fuite de la ville dense versus centres anciens et versus grands ensembles.

La séduction pavillonnaire repose en ce sens sur de nombreuses raisons qui oscillent et se hiérarchisent de manière variable selon l'époque d'installation. Dans les motivations de l'accédant, l'argument patrimonial est indéniable, l'argument familial intervient également, l'argument environnemental n'a cessé de se renforcer. La ville des années 70 lui a donné raison : les quartiers anciens s'y dégradent, les nouveaux quartiers d'habitat collectif, social ou non, n'ont que le confort à proposer dans une atmosphère d'immenses chantiers qui peinent à s'achever. La ville 
des années 70 est, du centre envahi par le trafic automobile à sa périphérie où alternent les zones d'habitat et les zones industrielles, une ville repoussoir.

La généralisation de l'automobile facilite une installation en retrait de la ville. Les villagescommunes proches offrent des terrains constructibles à des prix qui rendent l'accession à la propriété envisageable et un cadre de vie qui fait vibrer le souvenir nostalgique d'une ascendance rurale. Pour beaucoup, elle n'est pas si lointaine.

Mais la maison s'isole aussi parce que, dans ces premiers temps de la télévision, la ville se fait moins indispensable : la petite autarcie domestique du pavillon s'accommode bien de ces loisirs qui viennent à la maison et font baisser la fréquentation des "salles obscures ", puis de ces matchs de foot qui ne se limitent plus au soutien de l'équipe locale, depuis les tribunes du stade exposées au froid glacial des nocturnes d'hiver.

Nombre d'activités de la ville paraissent ainsi entrer dans l'appartement, prolongeant un mouvement de privatisation de la scène publique qu'avait inauguré le XIXe siècle en inscrivant les lieux de commodités ou d'aisance dans le logement, à l'instar de ce qu'avaient souhaité les grandes dames de l'aristocratie, annonçant ainsi la fin proche des " reines du lavoir" .

Ce mouvement est pourtant paradoxal, puisqu'en privatisant des activités autrefois dévolues au monde du dehors, il renforce certes les intimités de l'intérieur, fabrique cette famille moderne du modèle bourgeois, élargit le " colloque familial " en l'instruisant au-delà de la parole du père, mais il y fait aussi pénétrer plus amplement, en temps et en audience, les questions et les débats du monde public, y démocratise les spectacles autrefois réservés à des élites restreintes, y vulgarise bientôt les voyages lointains qui ne se donnaient jusqu'alors qu'à être racontés ou lus. La boite magique fait entrer le monde dans la maison et son offensive n'a de cesse, par reality show et autre love story interreliés.

La maison a en apparence moins besoin de la ville, bien qu'elle y puise ses ressources ; l'automobile familiale lui facilite l'accès aux biens de consommation encore largement acquis en ville (leur consistance matérielle appelle un déplacement que la vente par correspondance peut économiser, au risque d'une surprise quant à la qualité du produit) et le congélateur va pouvoir restreindre la fréquence de ces déplacements ; la diffusion exponentielle des matériels audiovisuels met à portée de maison le produit de spectacles et de concerts qui trouvent aujourd'hui, dans les box d'enregistrements, une autre voie, moins spontanée, pour lisser des interprétations dont l'écoute répétée finit par ne plus supporter les imperfections du " direct ".

La maison est en ce sens paradoxale : à la fois plus privée, car plus retirée, elle est en même temps plus publique car elle a capté des activités qui se donnaient anciennement en des lieux publics. Les scènes publiques qu'elle s'est annexées, au moyen d'appareils audiovisuels principalement, font pénétrer dans l'univers domestique des formes culturelles ou politiques, dont la gamme va des expressions les plus arrêtées (l'enregistrement) aux plus spontanées (le match " en direct "), des événements les plus graves du monde aux manifestations les plus ludiques, diffusées par des présentateurs qui peuvent devenir des locuteurs " médiatiques ".

Mieux, dès lors que leur présence n'est même plus simplement virtuelle et lointaine, du fait du développement de l'interactivité encouragée par la radio et la télévision, ils peuvent devenir des "interlocuteurs " (Dolto en a peut-être inauguré le genre), et se faire les confidents d'anonymes qui, depuis leur maison, dévoilent sur les antennes des " drames " privés ainsi libérés d'un enfermement domestique pesant. 


\section{Le leurre de l'écart}

L'isolement de la maison est en réalité un leurre, mais on conviendra que les illusions peuvent aider à vivre.

Cependant le travail du chercheur consiste à ne pas être dupe de ce leurre ; à en prendre la mesure, de telle sorte qu'il donne à comprendre combien la société peut " se raconter des histoires ". A ce stade, il me faut aussi insister sur les belles histoires que se racontent aussi parfois- les chercheurs en ne retenant de la maison que la version poétique dans laquelle nous a bercé Bachelard. L'avertissement est donc donné : je parlerai dans la suite de choses particulièrement triviales, -au risque d'abîmer le rêve pavillonnaire- de raccords, de fils, de tuyauteries et d'entrailles.

Car c'est par eux que la maison est raccordée à la ville, tout en s'ingéniant le plus possible à nous les faire oublier, pour dire en quoi elle s'est libérée des mitoyennetés qui l'étouffaient. L'existence de ces réseaux, qui ont mauvaise presse, montre en même temps que la maison ne peut pas vivre sans la ville, même si elle s'est implantée hors de la ville pour satisfaire le désir de coupure de son habitant.

La maison du péri-urbain est justement le compromis de cette installation "rurbaine " qui cherche l'environnement le plus représentatif de la non-ville, c'est-à-dire " rural ". Dans les années 70, le processus d'accession reprendra de l'ampleur sous la férule du Ministre Chalandon. Le promoteur des " chalandonnettes " se plaisait à dire comment la maison isolée allait venir à bout de la vieille " contradiction ", tant soulignée au XIXe siècle, " entre ville et campagne ". En ces franges de campagne " mitée " par l'urbanisation, la ville perdra donc définitivement cette limite franche de ville close à laquelle les grands ensembles prétendaient offrir, contre " l'anarchie pavillonnaire ", le dernier rempart.

La pression accédante favorise dès lors le délaissement des activités agricoles, d'inégale valeur selon les localités, au profit d'une urbanisation qui se dit " limitée " dans la rédaction des règlements -mais qui laisse filer les constructions au gré de ventes multipliées et mésuse d'une structure parcellaire agraire non préparée à l'urbanisation. Les terrains excèdent souvent, par la surface, tant celle requise dans le Plan d'occupation des sols que celle dont l'habitant a potentiellement besoin. Leur desserte est problématique, conditionnée par des servitudes de passage qui ne facilitent ni leur accessibilité depuis la voie publique, ni leur alimentation en eau ou en électricité, et peuvent engendrer les litiges de voisinages.

$\mathrm{Au}$ fur et à mesure que ces villas s'additionnent le long des chemins vicinaux, le nombre des boîtes à lettres plantées au croisement des voies asphaltées et des chemins de terre ne cesse de croitre : dans ces écarts urbains la numérotation des demeures dit bien leur appartenance à la ville, et les quatre chiffres des plus extrêmes implantations $(3451,3452 \ldots)$ montrent d'une certaine façon que la construction des maisons a débordé la viabilisation des chaussées qui les desservent. La fréquence des circulations qui les empruntent contribue à les dégrader encore et suscitera bientôt les récriminations de ces propriétaires. Ils paient une lourde charge foncière sans que la commune ne s'occupe de l'état de ces routes à la hauteur de leur impôt. Elle reste par ailleurs scandaleusement indifférente à leurs appels répétés en faveur de la viabilisation des chemins ou des passages nouveaux -qu'ils ont ouverts avec des véhicules $4 \times 4$ leur permettant d'affronter et d'aggraver des ornières à chaque orage plus profondes. 


\section{Une maison-territoire}

Au fond, chacun de ces nouveaux foyers, souvent détracteurs des lotissements, autres écarts plus organisés, a d'abord établi sa villa sur un terrain qu'il a choisi pour la générosité de sa surface, -lui apportant l'écart aux autres nécessaire à la " tranquillité " longtemps espérée. Mais aussi dans un paysage provençal dont il apprécie au plus haut point les lignes, les silhouettes et les couleurs. Il souhaite ardemment qu'après sa propre installation il restera ce même paysage, arrêté une fois pour toute, immuable, éternel, et qu'aucune autre construction ne viendra boucher le panorama qu'il découvre chaque jour à son réveil. Les péri-urbains du grand écart vivent comme leur propriété -sans en avoir la possession-, l'environnement de leur maison, comme une bastide sur l'étendue de ses terres, comme les châteaux des coteaux d'Aix-en-Provence en donnent encore l'image au cœur de leurs vignobles.

Les nouvelles constructions, qui peuvent procéder de la même entreprise d'accession, apparaissent dès lors pour les "déjà-installés" comme une atteinte à ce paysage. A défaut de maitriser sa production culturale, on a intégré avec conviction le discours de sa protection culturelle. La silhouette de la Sainte-Victoire participe de ces " hauts lieux " du pays d'Aix et le nec plus ultra de l'installation résidentielle consistera à bénéficier, depuis fenêtres et terrasses, d'une ouverture sur le profil triangulaire de cet anti-synclinal maintes fois représenté par Cézanne. Mais bien d'autres chaînes et collines, bois et falaises de calcaire participent de cet assemblage de reliefs naturels et de terres cultivées qui accompagnent la " villa " et la font valoir. Le village perché est aussi une de ces figures du paysage provençal, encore plus artialisée, qui fait office de référence dans la construction du territoire élargi de la maison. D'ailleurs on le regarde de loin, ce village, plus qu'on ne le fréquente : on le sait présent dans le paysage, comme fondu en lui, et il sert de puissant identifiant pour se dire de cet endroit ou pour situer son adresse et, à l'adresse des autres, la " villa " où l'on réside.

Ainsi le territoire de la " villa " se définit par au moins deux cercles concentriques successifs. Le premier est inscrit dans les limites de la "propriété privée ". Elles seront d'autant moins nettes que la superficie de son assise foncière sera plus étendue, mais leur rappel, sur les chemins, aux moyens de cercles rouges d'interdiction ou de menaces canines, sera d'autant plus insistant que la propriété se confond dans la nature. Le second cercle est défini par l'horizon visible depuis la " villa ", et dont la figure idéale est celle d'un paysage totalement " dégagé ", que l'on peut balayer à $360^{\circ}$ autour de la construction, et qui ordonne dans ce panoramique les vues successives des chaînes, des collines et du village.

\section{L'hinterland de la maison}

Mais s'arrêter là nous donnerait la traduction d'une autarcie sédentaire qui ne correspond en fait qu'à une présence réduite du résident péri-urbain dans sa demeure : ce repli qu'il accomplit pleinement le dimanche. Cette retraite tant espérée peut aussi s'étendre au premier jour du weekend pour peu que les approvisionnements de la maison aient été réalisés antérieurement, au cours de ces innombrables déplacements auxquels l'écarté doit se livrer pour son propre travail, la formation et les loisirs de ses enfants.

Ici se forment des couloirs de circulation et de distribution vers les destinations inscrites dans les principales polarités urbaines, celles qui concentrent les lieux d'occupations professionnelles et de services des membres de la maisonnée. Ces couloirs donnent à pratiquer des infrastructures routières et autoroutières dont on a expérimenté les avantages et les inconvénients, les zones embouteillées et les segments dangereux, tout un réseau avec lequel on s'est familiarisé. À la 
vitesse des autoroutes qui livrent, pour peu qu'on soit disponible, les grands traits du paysage, succèdent la circulation moins rapide et plus heurtée des routes secondaires, la traversée des villages longiformes formés au bas des villages perchés, l'arrêt opportun dans les commerces sauvages des bords de routes, et parfois, sur le chemin du retour vers la villa, une conduite buissonnière qui délasse du "stress " des sorties urbaines encombrées.

Ces couloirs de circulation construisent les territoires péridomestiques et hypométropolitains des habitants du périurbain, un ensemble de lieux et d'itinéraires aussi variés que tendent à le devenir les centres d'intérêt et les "expériences " des individus composant le groupe familial, jeunes et moins jeunes, plus au moins autonomes dans leurs facultés de déplacement. Ces territoires se superposent et s'entrecroisent, s'inscrivent ou sortent d'un territoire familial commun, selon des rythmes temporaires plus ou moins rapprochés et réguliers ; ils participent d'un territoire flou, plus vaste que les territoires personnels : sinon invisible, du moins difficilement lisible, il entre dans la construction de la ville désurbanisée. Il échappe de toute façon à l'horizon restreint du paysage formé par la vie résidentielle en villa et lie cette dernière, en fréquence variable, à la ville et à ses diverses polarités, sources d'emploi, de formation, d'approvisionnement et de loisirs.

Et le moyen de cette liaison est précisément, à défaut d'autres modes aussi performants, l'automobile, les automobiles, qui ont rendu possible la séparation -à ce point distante- de la résidence et du travail. Cette distance fait du couple villa / voiture, un système qui, dans sa version la plus radicale, ne peut accepter que l'une existe sans l'autre. Et c'est précisément la fréquence régulière, sinon quotidienne, de ces trajets dans les couloirs de circulation mille fois empruntés qui les instituent en territoires familiers.

\section{Entre territoire péridomestique et inscription métropolitaine}

L'habitant de la " villa " pratique par conséquent deux types d'inscription urbaine.

La première, la plus étroite, est engendrée par la fréquentation régulière de lieux situés, en deçà et un peu au-delà de l'horizon visible de la " villa ", dans une certaine proximité de cette dernière, à portée de " coup de vélo ", et plus souvent de " coup d'auto ", souvent hors des limites d'une commune que l'on identifie bien plus par son (ses) noyau(x) villageois que par ses " frontières ". Ce territoire est non seulement bien connu, parce que pratiqué avec régularité, mais aussi fortement approprié comme territoire de référence résidentielle positivé. Il s'y est construit une appartenance "pour soi ". Tantôt elle consistera, pour ceux que nous appelons les " locaux " -les plus anciennement établis des péri-urbains-, en la reconstruction d'un héritage transmis : de villégiature (les "cabanons " marseillais agrandis) ou de communauté minière (les descendants de mineurs du bassin de Gardanne). Tantôt, pour d'autres, ceux que nous appelons les "colons " qui sont souvent venus plus récemment d'autres régions-, elle consistera en l'élaboration (récupération) hétéroclite d'une identité nouvelle faite d'attrait pour le pavillon, pour le paysage qui l'environne et lui donne sa (le met en) valeur, de références cultivées sur les " hauts lieux " de la Provence, sur l'art de vivre (d'habiter, d'inviter...) en cette belle région ensoleillée.

La seconde inscription territoriale, plus lointaine, plus parcellaire, est fondée bien davantage sur la fréquence des déplacements et la répétition des situations circulatoires que par une appropriation voulue des lieux traversés ou atteints. Cependant, dans ces mouvements et cette agitation incessants, une connaissance des couloirs de circulation se construit progressivement : elle se saisira de ci de là (en réciprocité : comme les marchands se sont saisis de la fréquence des passages pour installer de grandes zones telle que Plan de Campagne), d'occasions de détours vers les très grandes surfaces, mais aussi d'escapades ludiques (marchés provençaux, brocantes 
villageoises, foires aux vins, fêtes de la mer...) et de défis sportifs (ascension de la Sainte-Victoire, plongée...) pour épaissir sensiblement l'étroitesse des couloirs ; elle en amplifiera la valeur utilitaire habituelle pour la transporter vers une appropriation territorialement et culturellement plus positive.

Un territoire de dimension métropolitaine se forme ici, qui entre aussi dans la représentation des périurbains ; ceux-ci contribuent à lui façonner -non seulement par leur établissement pavillonnaire, mais aussi par la multiplication de leurs déplacements-, le caractère d'un territoire continu, en regard de leur vécu quotidien, alternant de manière dense les réseaux de routes et autoroutes, de villages anciens perchés, de lotissements et de semis de pavillons auxquels ils participent directement, de terres cultivées et friches agricoles, de massifs rocheux impropres aux constructions. Mais pour ces périurbains il demeure un territoire " malgré eux ", vis-à-vis duquel ils n'entretiennent qu'une appartenance " en soi ", pour paraphraser ce que disait Marx des formes de consciences sociales.

Le territoire péridomestique est celui des pratiques les plus régulières d'approvisionnement alimentaires et de loisirs, mais les unes et les autres peuvent ponctuellement s'évader vers des franges extrêmes de l'aire métropolitaine (grands centres commerciaux, arrière-pays, bord de mer...). Les achats alimentaires, pour ce qui les concerne, sont vécus sur le mode de la " corvée ", accomplis plutôt une fois que deux dans la semaine, la plupart du temps par la mère de famille, et plutôt dans des moyennes surfaces qui ponctuent les différentes communes situées entre Aix et Marseille que dans les très grandes surfaces. La fréquentation de ces dernières est plus espacée, et en même temps plus ludique, car motivée par ce qu'il est convenu d'appeler l'"équipement de la personne et de la maison".

La seule recherche d'un produit très spécifique pour l'activité d'un passionné de bricolage ou de jardin, par conséquent entièrement dédiée à la "production continue" de la " villa ", peut alors favoriser, seul, en couple ou en famille, un périple métropolitain qui donne ou non d'autres occasions d'achat : pendant que Monsieur fait ceci, Madame fait cela. D'autres fois le seul concerné par l'achat n'hésitera pas à aller, le même jour, d'est en ouest de l'étendue métropolitaine pour trouver le produit dont il a besoin. D'autres fois encore, la sortie entre personnes du même genre, la mère avec la fille, ou des copines, sera préférée à la sortie familiale pour des évasions ludiques dont l'autre sexe n'a que faire (" lèche-vitrine ").

Les loisirs, pratiqués sur le mode individuel (ballade à pied, en vélo...) ou associatif, privilégient eux aussi la proximité des équipements. Mais, dès qu'ils concernent les enfants, ils ont tendance à s'écarter vers des activités plus éloignées, parce que très spécialisées (équitation, piscine, plongée, sports martiaux...).

\section{Des territoires communs aux territoires personnels}

On aura déjà noté que ces différents territoires ne sont jamais exactement les mêmes pour les différents membres de la famille.

Le travail des parents, la plupart du temps l'activité professionnelle du père, dessinent l'extrémité du territoire quotidien, quand elle ne se prolonge pas, depuis Marignane, par une envolée vers la capitale, ou des capitales encore plus lointaines.

Les destinations des enfants, quant à elles, n'ont cessé de repousser leur éloignement, en même temps qu'ils franchissaient les paliers successifs de leur scolarisation. De maternelle en collège, la 
densité des équipements communaux ou intercommunaux s'amoindrit et oblige au changement des modes de transports. Déjà fort problématique en maternelle et en primaire pour les familles les plus écartées -ce qui rend nécessaire le remplacement de la marche à pied par les " deux roues "-, le transport vers le collège, puis vers le lycée devient un " casse-tête ". S'y entremêlent les contraintes d'un lever matinal et de rentrées tardives, la gestion des emplois du temps parentaux, la recherche des services réciproques entre voisins de résidence ou de classe, les inquiétudes sur les dangers d'utilisation des cycles ou des transports collectifs, la préoccupation des attentes oisives, des risques " d'entraînement " aux pratiques délinquantes...

Les parents, mais surtout les mères, se font alors une obligation morale d'assumer ces transports que la ville étalée n'a pas su ni pu préparer pour l'urbanisme dispersé des " villas ". Le phénomène des " mamans-taxis " prend dès lors tout son essor. Pourtant l'inconvénient de cette charge sera bientôt muté en avantage, car en intégrant cette pratique dans sa condition de périurbain, on exploitera au maximum l'inévitable recours à l'automobile pour élargir le choix des établissements scolaires -à travers leurs filières rares (musique, langues...), leur réputation et leur excellence supposées ou avérées-, "quitte à faire quelques kilomètres de plus " et au risque d'encombrer ou d"' empoisonner " un peu plus les artères de la ville et les bronches des urbains pédestres.

$\mathrm{La} "$ carte scolaire " -qui territorialisait le rapport entre la résidence et le système scolaire républicain et les fondait sur le couple proximité / égalité-, vole en éclats sous les coups de boutoir d'une liberté d'usage de l'automobile personnelle mise au profit d'une société plus inégale et de territoires plus ségrégés. On se défausse alors des règles administratives d'inscription scolaire par les procédés les plus légitimes comme par les petites tricheries les plus mesquines et on érige une nouvelle norme pratique de la consommation domestique et urbaine qui pourra associer, par la médiation de l'automobile, les prestiges cumulés d'une scolarisation hypercentrale et d'une installation résidentielle hyperpériphérique.

Un cadre de pratiques s'est ainsi mis en place qu'on appellera, par pléonasme hétérolinguistique, " domestico-économique " : il pose le travail comme une donnée de départ incontournable et la mise à l'écart de la " villa " comme un choix contraint (décidé dans le compromis entre le désir de campagne, la distance-temps maximale au travail, les moyens financiers et les disponibilités foncières). Dans ce contexte, le dévolu sur les établissements scolaires intervient en troisième lieu et s'inscrit dans un marché de la formation qui maximise les avantages-inconvénients de la périurbanisation (acquis de la mobilité, déficit des équipements scolaires locaux) pour en tirer le meilleur profit en termes de capitalisation culturelle des enfants.

Rien n'obligeait à faire ce choix du "meilleur établissement" en dépit d'un plus grand éloignement, mais l'incorporation du réflexe automobile pousse à l'adopter. Son caractère de "libre" choix invite à dès lors à inscrire cette destination et le trajet qui y conduit dans la construction territoriale de l'habitant du péri-urbain, car il est pertinent de définir comme territoire de cet habitant, hors toute considération renvoyant aux découpages administratifs et institutionnels, un ensemble de lieux structurés selon une certaine cohérence (évidente ou apparente) en regard d'un projet de vie formé pour soi-même ou sa famille.

\section{Tensions}

Et pourtant la liberté de ce choix est bien relative, puisqu'il se heurte à des impossibilités ou d'importants désagréments du fait de la "dépendance automobile " . L'offre scolaire paraît plus étendue avec la mobilité, mais comme pour d'autres activités extrascolaires, culturelles ou sportives, l'écart résidentiel astreint l'un des parents, presque toujours la mère, à multiplier les 
déplacements sans que sa disponibilité satisfasse à tout coup les attentes d'adolescents qui ne vivent pas leur retranchement champêtre sur le mode enchanté des parents.

Pour ces derniers, le projet d'accession a souvent été pensé lors de l'acte de fondation familiale, réalisé au moment où les enfants ont pu profiter du petit territoire aéré et protégé du jardin familial. Devenus plus grands, si les parents continuent de nourrir ce modèle en invitant les copains de leurs enfants pour conserver un œil sur leurs rejetons, les adolescents aspirent bientôt à des rencontres sociales entre jeunes, hors du contrôle parental, dans des espaces publics les garantissant d'un anonymat que ne leur autorise nullement une bienveillance parentale à peine moins lourde que ne l'étaient les anciens voisinages villageois.

Ils réclament dès lors soit un vélomoteur -ou mieux un scooter-, qui n'est pas obtenu sans beaucoup de réticence, soit -lorsqu'ils sont en mesure de conduire-, le prêt d'un véhicule parental (le second, "celui " de la mère), ou l'achat d'un troisième. Car là encore s'en remettre aux transports en commun se révèle une entreprise désespérée : leur fréquence et leur parcours, laborieusement programmée et tracé pour desservir aux mieux les écarts résidentiels les plus retirés, ne peut laisser espérer au jeune qui aura recours à ce mode de déplacement, un temps d'activité sur le lieu de destination à peine plus long que celui qu'il aura consacré à y être transporté.

\section{De la maison-monde à la ville-maison}

Car cette multiplicité de désirs suscités par la vie contemporaine, encore plus radicalisés lorsqu'il s'agit des attentes ludiques et culturelles, est loin de pouvoir se satisfaire d'une offre qui trouve pourtant sa traduction domestique interne dans la multiplication des appareils électroniques, domestiques et statiques ou bien personnels et portables.

Cet élargissement des possibilités de la personne, rendant plus autonomes, plus indépendants les membres de la famille, les jeunes générations des plus vieilles, les femmes des hommes, pouvait laisser croire à certains prophètes l'advenue du "cocooning ". En réalité, cette possibilité de s'isoler dans des mondes plus étanches aux proches (les parents qui sont là dans la maison), mais en même temps plus partagés par des affinitaires lointains (en particulier comme internautes), attise aussi l'envie de côtoyer cette multitude dispersée de semblables, que seule la ville dense, en des lieux publics et en des figures humaines -qui parlent surtout par leurs signes extérieurs d'allure, de coiffures et de vêture-, permet de croiser dans la connivence de l'anonymat, et d'y mettre en représentation son apparentement.

Ce désir de ville, donc de rencontre et d'urbanité, plus vivace que jamais, se concrétise dès lors dans la "sortie " en ville. La ville s'entend ici dans une acception large, mais dans une référence à l'urbain marquée par l'association matérielle et sociale de la densité et de l'urbanité, des espaces et des gens. Il s'agit donc ici autant des centres villes anciens que de ces nouvelles centralités surgies à la périphérie des villes. Cette attente d'urbanité a favorisé depuis quelques décennies la reconquête piétonne des centres villes. Cette reconquête traduit en même temps le déplacement de la consommation de masse du nécessaire à l'accessoire, de l'achat au meilleur prix vers la "petite folie " d'un jour, tendant à "démocratiser" le luxe, sinon pour en faire son quotidien, du moins pour en faire le prétexte d'un regard, d'une envie qu'aucune morale de la "restriction" ou de l'"économie" n'est plus à même de retenir. Les centres villes y trouvent une nouvelle jeunesse, et les grandes surfaces des périphéries se sont tôt empressées de créer des rues intérieures abritées alignant "boutique" sur "boutique", cette forme commerciale reprise des centres anciens. Les zones commerciales redoublent et séparent ainsi l'immense aire de stationnement des véhicules, 
d'une galerie marchande qui transporte à l'intérieur (privé) du centre commercial le décor, extérieur et public, d'une rue urbaine insuffisamment accessible dans le centre ancien.

Si ces grands centres sortent les parents de la "corvée" des commissions, en y associant un intermède ludique (et encore bien plus les rues piétonnes des centres-villes), toutes ces centralités constituent pour les adolescents un gisement d'opportunités innombrables de rencontres, de spectacles, de jeux, de tentations d'achat, d'essayages, de pratiques du corps à corps urbain que la débauche virtuelle dépensée dans la "villa " isolée ne parvient pas à satisfaire. La ville est alors pour eux comme une autre maison, plus habitable que l'écart où le désir de tranquillité de leurs parents les a cantonnés. Trop dépendants du bon vouloir de ces derniers, lorsqu'il s'agit de leur donner l'accès à ces centralités denses en les y transportant, ces jeunes vivent souvent l'évasion champêtre de leurs géniteurs comme des temps et des lieux d'ennui lancinant, malgré la quantité d'appareils de communication qu'ils ont pu y installer.

\section{Pour conclure}

Il est bien possible que la " villa " écartée ne soit qu'une réponse circonstancielle, moins structurelle que ne pourrait le laisser penser la référence au modèle américain : une réaction à l'évolution de la ville, marquée au XIXe siècle par sa densification intra muros et au XXe siècle par l'envahissement de la "reine automobile", dégradations et altérations qui n'ont pu qu'exacerber les tentatives d'évasion permises par la vulgarisation de la voiture et du pavillon.

Mais en réalité tout montre que cet écart de la " villa " n'est qu'illusion, artifice de distance. L'installation en campagne masque mal en effet la densité des liens de cette habitation avec la ville, par réseaux plus ou moins visibles, terrestres, souterrains ou aériens, interposés / entrecroisés. Ces réseaux, une somme de véhicules réels ou virtuels les parcourt en tous sens pour transporter, entre la " villa " et des lieux innombrables de la ville, des corps, des matières et des messages.

Les territoires de la " villa " que nous nous sommes efforcés d'identifier dans les pratiques de déplacement multipliées de ces faux-isolés montrent l'intensité de ces liens à la ville, l'étendue et la diversité de leur couverture métropolitaine entre territoires péridomestiques et grand territoire interurbain. Cette inscription urbaine du pavillon participe bien du phénomène de la métropolisation en dépit d'un écart pensé en réaction à la dégradation de la ville ancienne ou à la froide mécanisation ("machinisation") du logement moderne.

L'étalement des franges urbaines qui en résulte fait également apparaitre, par les tensions qu'il engendre dans le transport fortement individualisé de ces corps, mais aussi par les difficultés dans l'accessibilité aux lieux de véritable urbanité que sont les centres denses, que l'écartement spatial n'est sans doute pas la meilleure solution pour cette ville-maison habitable -réédition moderne du vieux projet d'Alberti pour qui la ville était une grande maison et la maison une petite ville-, et à laquelle nous semblent aspirer des fractions sans cesse plus larges de la société.

L'habitabilité avait comme déserté la ville pour trouver refuge dans le logement, et les établissements (settlement), ces " véritables unités territoriales " ont unilatéralement cédé aux exigences d'une mobilité accrue, impliquant qu'ils se plient plus volontiers à l'irruption des engins qu'à l'accueil des personnes, qu'ils se soucient plus des machines que des corps. Aujourd'hui différents indices (le partage de l'espace public, l'attention portée aux paysages...) montrent que l'habitabilité de la ville, c'est-à-dire sa capacité à assurer la protection et l'accueil des corps, comme "une grande maison ", participe des attentes sociales. 
Références bibliographiques:

Augé, M. (1992), Non-lieux. Paris : Le Seuil.

Bachelard, G. (1955), La poétique de l'espace. Paris : PUF.

Bauer, G., Roux J.-M. (1976), La rurbanisation ou la ville éparpillée. Paris : Le Seuil.

Bonnin, Ph. (1994), "L'habitation, modalités de l'existence sociale", in Les hommes, leurs espaces et leurs aspirations. Mélanges en l'honneur de Paul-Henry Chombart de Lawne. Paris : L'Harmattan.

Bonnin, Ph., de Villanova, R. (dir.) (1999), D'une maison l'autre, parcours et mobilités résidentielles. Grane : Créaphis.

Choay, F. (1981), La rìgle et le modèle. Paris : Le Seuil.

Cuturello, P., Godard, F. (1982), Familles Mobilisée s: Accession à la propriété et notion d'effort des ménages. Paris : Plan Construction.

Donnadieu, P. (2000), "Campagnes et natures urbaines ", in Paquot, Th., Lussault, M., Body-Gendrot, S. (sous la dir.), La ville et l'urbain, l'état des savoirs. Paris : La Découverte.

Dubet F., Martucelli D., (1998), Dans quelle societé vivons-nous? Paris : Le Seuil.

Marié, M., Viard, J. (1988), La campagne inventée. Arles : Actes Sud.

Dupuy G., (1999), La dépendance automobile. Symptômes, analyses, diagnostic, traitements. Paris : Anthropos.

Giedion, S. (1980), La mécanisation au pouvoir. Paris : CCI, Centre G. Pompidou.

Le Breton, D. (2000), Anthropologie du corps et modernité. Paris : PUF.

Lévy, J. (2000), "La France urbaine dans l'Europe des villes", in Paquot, Th., Lussault, M., Body-Gendrot, S. (sous la dir.), La ville et l'urbain, l'état des savoirs. Paris : La Découverte.

Mauss, M. (1950), Sociologie et anthropologie. Paris : PUF.

Orfeuil J.-P. (1994), Je suis l'automobile. La Tour d'Aigues : L'Aube.

Peillon, P. (2001), Utopie et désordre urbains; Essai sur les grands ensembles d'babitation. La Tour d'Aigues : éditions de l'Aube.

Perrot, M. (1987), Histoire de la vie privée, de la Révolution à la Grande Guerre. Paris : Le Seuil.

Pinson, D. (1988), Du logement pour tous aux maisons en tous genres. Paris : Recherches, Plan construction et habitat, MEL.

Pinson, D. (1999) "De la mobi-stabilité ", in Urbanisme n³06, p. 84-89.

Pinson, D., Thomann, S. (2002), La maison en ses territoires. Paris : L'Harmattan.

Raymond, H. et M.-G., Haumont A. et N. (1964), L'habitat pavillonnaire. Paris : CRU.

Singly de- F. (1996), Le soi, le couple et la famille... Paris : Nathan.

Verret, M. (1978), L'espace ouvrier. Paris : A. Colin.

Wiel, M. (1999), La transition urbaine. Liège : Mardaga 